\title{
Síntesis de Microcápsulas de Poliurea a partir de Aminas Renovables, Mediante Doble Emulsificación
}

\author{
Paula Mazo, Luis A. Rios, Gloria Restrepo \\ Departamento de Ingeniería Química, Universidad de Antioquia
}

\begin{abstract}
Resumen: En este trabajo se realizó la microencapsulación de un perfume comercial mediante una doble emulsificación, la coraza de poliurea fue sintetizada por la reacción de Lisina con dos diisocianatos comerciales, uno aromático y otro alifático. En la síntesis se evaluó el efecto que tiene la relación molar amina:diisocianato y el tipo de emulsificante. Se optimizó el tamaño de partícula utilizando un diseño factorial $3^{2}$ y análisis de superficie de respuesta, las variables fueron: cantidad de alcohol polivinílico y la relación de fase dispersa a fase continua. Las microcápsulas se caracterizaron mediante: análisis de calorimetría diferencial de barrido (DSC), espectroscopía infrarroja (IR), microscopía electrónica de barrido (SEM) y tamaño medio de partícula. La doble emulsificación permite un mayor rendimiento de encapsulación del perfume, las micropartículas presentan un menor tamaño de partícula cuando: se emplea diisocianato aromático, un aumento de coloide protector (PVA) y una disminución de la fase dispersa.
\end{abstract}

Palabras-claves: Microencapsulación, poliurea, lisina, poliglicerol poliricinoleato.

\section{Synthesis of Polyurea Microcapsules from Renewable Amines by Double Emulsification}

\begin{abstract}
This paper reports on the microencapsulation of a commercial perfume by means of a double emulsification, where the polyurea shell was synthesized by the reaction of lysine with two commercial di-isocyanates (aromatic and aliphatic). In the synthesis the factors evaluated were the amine:di-isocyanate molar ratio and the type of emulsifier. The particle size was optimized using a $3^{2}$ factorial design and response surface analysis, with the following variables: amount of polyvinyl alcohol and the relationship of the disperse phase to continuous phase. The microcapsules were characterized using differential scanning calorimetry analysis (DSC), infrared spectroscopy (IR), scanning electron microscopy (SEM) and mean particle size. The double emulsification allows for a greater yield in the encapsulation of perfume. Furthermore, the microparticles have a smaller particle size when the aromatic di-isocyanate was used, also leading to an increase in the protective colloid (polyvinyl alcohol (PVA)) and reduction of the disperse phase.
\end{abstract}

Keywords: Microencapsulation, polyurea, lysine, polyglycerol polyricinoleate.

\section{Introducción}

La poliurea (PUREA) es un polímero muy atractivo para aplicaciones en recubrimientos: se forma por la reacción in situ de poliisocianatos con poliaminas, y sus propiedades mecánicas pueden ser controladas dependiendo de la estructura química, el peso molecular de los componentes y de la estequiometria de la reacción, la selección de los reactivos se hizo con base en las propiedades deseadas, el costo y la utilización de recursos renovables y biodegradables. Los isocianatos alifáticos son muy empleados para aplicaciones que requieren altas prestaciones y alta resistencia a la luz, porque retardan la oxidación y el amarillamiento, los isocianatos aromáticos son más reactivos y más económicos que los alifáticos. En nuestro caso la amina seleccionada es un aminoácido de fácil adquisición y económico (Lisina) obtenido desde fuentes renovables.

Las poliureas son materiales segmentados con una fase dura (isocianato) dentro de una matriz flexible y presenta alta dureza debido a la gran cantidad de puentes de hidrogeno intermoleculares ${ }^{[1]}$.

La principal aplicación de la poliurea es para la microencapsulación en las áreas: farmacéutica, industria cosmética, alimentaria y agroquímica, para reducir la contaminación ambiental generada por los plaguicidas, ya que al encapsular los ingredientes activos de estos productos se puede controlar su liberación, aumentando así su eficacia y persistencia, y además, reduciendo las cantidades liberadas al ambiente ${ }^{[2]}$, papeles de copia sin carbó $^{[3]}$, microcápsulas multicapa, que mejoran sus propiedades de resistencia mecánica ${ }^{[4,5]}$, microencapsulación de catalizadores para aplicaciones en síntesis química ${ }^{[6,7]}$, microencapsulación de fosfato de amonio como retardante a la llama en aplicaciones textiles ${ }^{[8]}$, recientemente la microencapsulacion de materiales de cambio de fase ha sido estudiada para aplicaciones en campos de energía térmica y aires acondicionados de edificios, materiales de aislamiento térmico, fibras térmicas adaptables, etc. Los materiales de cambio de fase pueden absorber, almacenar y liberar grandes cantidades de calor latente en un rango definido de temperatura mientras el material cambia de fase o estado ${ }^{[9-11]}$ y la microencapsulación de líquidos reactivos ${ }^{[12]}$.

La microencapsulación es un proceso de recubrimiento de sustancias sólidas, líquidas o gaseosas con diferentes materiales, con miras a obtener partículas sólidas de tamaño micrométrico. Los productos resultantes de este proceso tecnológico reciben el nombre de "micropartículas", "microcápsulas" o "microesferas", las cuales se diferencian en su forma y estructura interna; sin embargo, todas presentan como característica común el poseer un tamaño de partícula inferior a $1,0 \mathrm{~mm}^{[13]}$.

Las microcápsulas con perfume son muy utilizadas en el sector textil, obteniéndose tejidos funcionales de mayor valor agregado, nosotros las emplearíamos como relleno en un trabajo posterior para materiales de poliuretano (PU) obtenidos a partir de aceites vegetales, las cuales disminuirían el mal olor que presentan los PU y aumentarían las propiedades mecánicas ya que son muy compatibles con los mismos por las fuerzas intermoleculares que forman entre si (puentes de hidrogeno). 
Existen diversos métodos para la producción de microcápsulas. En general, estos métodos se pueden dividir en tres grupos ${ }^{[14]}$ :

- procesos físicos: secado por aspersión;

- procesos químicos: polimerización interfacial e inclusión molecular; y

- procesos fisicoquímicos: coacervación, liposomas y gelificación iónica.

En nuestro trabajo, seleccionaremos la polimerización interfacial para la obtención de las poliureas, porque es un método muy económico, el proceso es muy sencillo, necesita bajas temperaturas de reacción, es poco contaminante y tiene alto rendimiento para la microencapsulación de perfumes.

Polimerización interfacial ${ }^{[15]}$ :

En este proceso se produce la polimerización de un monómero en la interfase de dos sustancias inmiscibles, formando una membrana, que dará lugar a la pared de la microcápsulas. Para que ocurra este proceso debe considerarse las siguientes etapas:

1) Equilibrio iónico para el monómero en la fase acuosa;

2) Transporte del monómero de la fase acuosa y/o fase orgánica al sitio de reacción;

3) La reacción entre los dos monómeros; y

4) La separación de fases de las especies oligoméricas formadas y la formación de la película.

En este trabajo se propone optimizar mediante un diseño factorial y superficie de respuesta el tamaño de partícula y la eficiencia de la microencapsulación de un perfume comercial hidrofílico, con una coraza de poliurea, formada por la reacción de dos tipos de diisocianato uno aromático y otro alifático con Lisina un aminoácido obtenido desde fuentes renovables. Se pretende estudiar algunos factores que afectan la reacción como es el índice $\mathrm{NCO}: \mathrm{OH}, \mathrm{pH}$, tipo de emulsificante y método de emulsificación.

\section{Experimental}

\section{Reactivos y métodos}

Para las reacciones se emplearon los siguientes reactivos: Lisina comercial (Premex), Toluen diisocianato (TDI) grado comercial mezcla 80:20 (Huntsman), hexametilen diisocianato (HDI) grado comercial (Huntsman), alcohol polivinilico e hidroxietilcelulosa, grado comercial (Protoquimica), sorbitan monooleato (Span 80) marca Aldrich, glicerina comercial (Protoquimica), ácido ricinoleico $\left(\mathrm{OHv}=380 \mathrm{mgKOH} . \mathrm{g}^{-1}, \mathrm{Av}=180 \mathrm{mgKOH} . \mathrm{g}^{-1}, 0.35 \%\right.$ humedad $)$, obtenido después de saponificar e hidrolizar aceite de ricino, resina DOWEX 50X2 (Dow)

\section{Diseño experimental}

Síntesis y caracterización de Poliglicerol-Poliricinoleato (PG-PR)

La síntesis de este emulsificante se realiza modificando el método empleado por Wilson et al. $1998^{[17]}$, mediante tres policondensaciones consecutivas, utilizando calentamiento por microondas en un horno Samsung, con un controlador de temperatura y una termocupla tipo $\mathrm{J}$, con una variación de $\pm 1^{\circ} \mathrm{C}$. El horno presenta una potencia de $800 \mathrm{~W}$ y la utilizada fue de $100 \%$.

1. Se cargan $300 \mathrm{~g}$ de glicerol con $3 \mathrm{~g}$ de $\mathrm{NaOH}$ ( $1 \%$ en peso), en un reactor de vidrio con entrada de nitrógeno, agitador mecánico $\mathrm{y}$ vacio para extraer la mezcla agua + glicerina formada por la autocondensación. A una temperatura de $200{ }^{\circ} \mathrm{C}$ por 2 horas, el poliglicerol obtenido se neutraliza mediante agitación con una resina de intercambio iónico ácida DOWEX 50X2. El número de hidroxilos (ASTM D4274 - 05) es: 860.67 mgKOH.g ${ }^{-1}$;

2. Se condensa ácido ricinoléico mediante agitación mecánica y vacio constante para extraer el agua de la reacción, a
$180{ }^{\circ} \mathrm{C}$ y 4 horas. Se cuantificó el número de hidroxilos (ASTM D4274 - 05) y el valor ácido (ASTM D4662 - 03): $\mathrm{OHv}=113.56 \mathrm{mgKOH} . \mathrm{g}^{-1}$ y Av $=55 \mathrm{mgKOH} . \mathrm{g}^{-1} ; \mathrm{y}$

3. $160 \mathrm{~g}$ de poliglicerol y $120 \mathrm{~g}$ de poliricinoleato, se condensan mediante agitación mecánica y vacio constante, a $200{ }^{\circ} \mathrm{C}$ por 4 horas. El número de hidroxilos obtenido fue de $290.52 \mathrm{mgKOH} . \mathrm{g}^{-1}$ y el de acidez es de $5 \mathrm{mgKOH} . \mathrm{g}^{-1}$.

\section{Síntesis y caracterización de las microcápsulas de poliurea}

La microencapsulación se realiza mediante polimerización interfacial utilizando una doble emulsificación para minimizar la reacción entre el diisocianato y el perfume que contiene grupos donadores de hidrogeno como son hidroxilos, aminas, ácidos, entre otros, estas reacciones son indeseables porque deterioran la calidad del aroma y disminuyen la cantidad de isocianato requerida. El procedimiento se realiza en tres etapas:

1. Emulsificación W/O: el perfume se agrega lentamente a la mezcla del diisocianato con el emulsificante a temperatura ambiente y $500 \mathrm{rpm}$ por 3 minutos. Se evaluaran dos emulsificantes: SPAN $80(\mathrm{HLB}=4.3)$ y poliglicerol poliricinoleato $(\mathrm{PG}-\mathrm{PR})$ $(\mathrm{HLB}=1)$

2. Emulsificación ó suspensión $\mathrm{O} / \mathrm{W}$ : La fase oleosa formada en el primer paso se adiciona lentamente a la solución de coloide protector, a temperatura ambiente, $800 \mathrm{rpm}$ y 3 minutos de agitación mecánica. Como coloides protectores se evaluarán: alcohol polivinilico e hidroxietilcelulosa; y

3. Polimerización interfacial: La amina solubilizada en agua se adiciona lentamente a la mezcla de reacción, mediante agitación continua a temperatura ambiente y $800 \mathrm{rpm}$. El seguimiento de la reacción se realiza midiendo el $\mathrm{pH}$ y se suspende cuando este sea constante, se decantan, centrifugan y se lavan las microcápsulas con una solución de etanol al 30\% en volumen y se secan a $25^{\circ} \mathrm{C}$ por 24 horas.

Después de evaluar las mejores condiciones de reacción se realiza la optimización utilizando un diseño factorial $3^{2}$ y se optimiza mediante la metodología de superficie de respuesta. Los variables y niveles se especifican en la Tabla 1. La Tabla 2 muestra los diseños experimentales, donde los experimentos de 1 a 14 y 15 a 28 corresponden con los sistemas Lisina-HDI y Lisina-TDI, respectivamente; en ambos diseños se realizan cuatro repeticiones al centro para estimar la desviación estándar.

El análisis estadístico se realiza empleando el software StatGraphics 5.1, empleando una ecuación polinomial de segundo orden:

$$
z=\beta_{0}+\beta_{1} x+\beta_{2} y+\beta_{3} x^{2}+\beta_{4} y^{2}+\beta_{5} x y
$$

donde:

- $\mathrm{z}$ : variable de respuesta tamaño de partícula.

- $\mathrm{x}$ : variable independiente codificada $\%$ alcohol polivinilico.

- y: variable codificada correspondiente a la relación en peso de la fase dispersa a la fase continua.

- $\beta_{0}, \beta_{1}, \beta_{2}, \beta_{3}, \beta_{4}, \beta_{5}$, son los coeficientes respectivos del intercepto, lineal, cuadrática e interacción.

Tabla 1. Variables y niveles empleados en la preparación de las microcápsulas.

\begin{tabular}{lllll}
\hline \multirow{2}{*}{} & Variables & \multicolumn{3}{c}{ Niveles } \\
\cline { 3 - 5 } & & $\mathbf{- 1}$ & $\mathbf{0}$ & \multicolumn{1}{c}{$\mathbf{1}$} \\
\hline 1 & \%PVA & 0.5 & 1 & 1.5 \\
2 & Wd/Wc* & 0.25 & 0.50 & 0.75 \\
\hline
\end{tabular}

\%PVA: porcentaje en peso de alcohol polivinilico, en la solución acuosa. Wd/Wc: relación en peso de la fase dispersa a la fase continua. 
Tabla 2. Cantidades de reactivos para la obtención de las microcápsulas.

\begin{tabular}{|c|c|c|c|c|c|c|c|c|}
\hline & \multirow[b]{2}{*}{ Ensayo } & \multirow[b]{2}{*}{$\mathbf{x}$} & \multirow[b]{2}{*}{$\mathbf{y}$} & \multicolumn{4}{|c|}{ Diseño de experimentos } & \multirow[b]{2}{*}{ Perfume (g) } \\
\hline & & & & PVA (g) & Agua (g) & Lisina (g) & HDI (g) & \\
\hline \multirow{14}{*}{$\begin{array}{l}\bar{\theta} \\
\dot{1} \\
\dot{y} \\
\dot{y}\end{array}$} & 1 & -1 & -1 & 8.88 & 1766.48 & 100 & 143.84 & 200 \\
\hline & 2 & 1 & -1 & 26.63 & 1748.73 & 100 & 143.84 & 200 \\
\hline & 3 & 0 & 1 & 5.92 & 585.87 & 100 & 143.84 & 200 \\
\hline & 4 & 1 & 0 & 13.32 & 874.36 & 100 & 143.84 & 200 \\
\hline & 5 & 0 & -1 & 17.75 & 1757.61 & 100 & 143.84 & 200 \\
\hline & 6 & 1 & 1 & 8.88 & 582.91 & 100 & 143.84 & 200 \\
\hline & 7 & 0 & 0 & 8.88 & 878.80 & 100 & 143.84 & 200 \\
\hline & 8 & -1 & 1 & 2.96 & 588.83 & 100 & 143.84 & 200 \\
\hline & 9 & -1 & 0 & 4.44 & 883.24 & 100 & 143.84 & 200 \\
\hline & 10 & 0 & 0 & 8.88 & 878.80 & 100 & 143.84 & 200 \\
\hline & 11 & 0 & 0 & 8.88 & 878.80 & 100 & 143.84 & 200 \\
\hline & 12 & 0 & 0 & 8.88 & 878.80 & 100 & 143.84 & 200 \\
\hline & 13 & 0 & 0 & 8.88 & 878.80 & 100 & 143.84 & 200 \\
\hline & 14 & 0 & 0 & 8.88 & 878.80 & 100 & 143.84 & 200 \\
\hline \multirow{15}{*}{$\begin{array}{l}\vec{\theta} \\
\dot{1} \\
\dot{y}\end{array}$} & Ensayo & $\mathbf{x}$ & $\mathbf{y}$ & PVA (g) & Agua (g) & Lisina (g) & TDI (g) & Perfume (g) \\
\hline & 15 & -1 & -1 & 8.98 & 1787.86 & 100 & 149.21 & 200 \\
\hline & 16 & 1 & -1 & 26.95 & 1769.89 & 100 & 149.21 & 200 \\
\hline & 17 & 0 & 1 & 5.99 & 592.96 & 100 & 149.21 & 200 \\
\hline & 18 & 1 & 0 & 13.48 & 884.94 & 100 & 149.21 & 200 \\
\hline & 19 & 0 & -1 & 17.97 & 1778.87 & 100 & 149.21 & 200 \\
\hline & 20 & 1 & 1 & 8.88 & 582.91 & 100 & 149.21 & 200 \\
\hline & 21 & 0 & 0 & 8.98 & 889.44 & 100 & 149.21 & 200 \\
\hline & 22 & -1 & 1 & 2.99 & 595.95 & 100 & 149.21 & 200 \\
\hline & 23 & -1 & 0 & 4.49 & 893.93 & 100 & 149.21 & 200 \\
\hline & 24 & 0 & 0 & 8.98 & 889.44 & 100 & 149.21 & 200 \\
\hline & 25 & 0 & 0 & 8.98 & 889.44 & 100 & 149.21 & 200 \\
\hline & 26 & 0 & 0 & 8.98 & 889.44 & 100 & 149.21 & 200 \\
\hline & 27 & 0 & 0 & 8.98 & 889.44 & 100 & 149.21 & 200 \\
\hline & 28 & 0 & 0 & 8.98 & 889.44 & 100 & 149.21 & 200 \\
\hline
\end{tabular}

La caracterización de los reactivos y las microcápsulas, se realiza mediante: espectroscopía IR espectrofotómetro marca Perkin Elmer y la morfología de la superficie fue observada en un microscopio de escaneo de electrones (scanning electron microscope. SEM) Hitachi S-520. Se realizó análisis de calorimetría diferencial de barrido (DSC) y análisis termogravimétrico (TGA) empleando un equipo TA Instrument a una velocidad de calentamiento de $10{ }^{\circ} \mathrm{C} / \mathrm{min}$ en atmosfera de nitrógeno y el tamaño medio de partícula se determinó en un equipo MASTERSIZER 2000.

\section{Resultados y Discusión}

\section{Síntesis de Poliglicerol-Poliricinoleato}

Este emulsificante es hidrofóbico, insoluble en agua, de alta viscosidad, con un color café oscuro. En la Figura 1 se muestran los espectros IR de PG, PR y PG-PR, productos de las tres policondensaciones. El espectro IR de PG-PR, muestra sus señales características: $3388.9 \mathrm{~cm}^{-1}$ estiramiento $\mathrm{OH}, 2930.3$ y $2860.4 \mathrm{~cm}^{-1}$ estiramientos $-\mathrm{CH}$ - alcano, $1737.58 \mathrm{~cm}^{-1}$ estiramiento $-\mathrm{CO}$ - del ácido ricinoleico, $1645 \mathrm{~cm}^{-1}$ estiramiento $\mathrm{C}=\mathrm{C}, 1457.6$ y $1339.5 \mathrm{~cm}^{-1}$ flexiones simétricas de los grupos $\mathrm{CH}_{2}$ y $\mathrm{CH}_{3}$ respectivamente, $1238.9,1107.7$ y $1046.5 \mathrm{~cm}^{-1}$ corresponden con estiramientos de -C-O- de ácido, ester y eter.

\section{Síntesis de microcápsulas estudio de variables}

La distribución y tamaño de partícula son parámetros importantes porque permiten determinar la homogeneidad y dimensiones, estimar la eficiencia de formación y lograr controlar el proceso de microencapsulación de las mismas. Se requieren partículas de un tamaño adecuado (50 a $100 \mu \mathrm{m})$ para facilitar su proceso de separación, que sean imperceptibles al tacto y evitar la aglomeración de las mismas.

Se evaluaron el efecto de diversas variables en la obtención de las micropartículas los cuales son:

\section{Seguimiento de la reacción en términos del pH}

La molécula de lisina tiene dos grupos amino y un ácido carboxílico, presenta tres valores de pKa: 2.2, 9.1, 10.5. Para valores de $\mathrm{pH}$ por encima de 10.5 , los grupos amina están libres $\left(\mathrm{NH}_{2}\right)$ y el ácido está como carboxilato ( $\left.\mathrm{COO}^{-}\right)$, por lo tanto se espera que la reacción con los grupos isocianato se de más fácilmente ${ }^{[16]}$.

El valor de $\mathrm{pH}$ inicial fue ajustado a 12 utilizando una solución $1 \mathrm{M}$ de $\mathrm{NaOH}$, y fue medido en el transcurso de la reacción para determinar el avance y finalización de la misma como se muestra en la Figura 2. El tiempo total de reacción para el consumo de la amina fue de aproximadamente 5 minutos en el caso de HDI y 2 minutos para el TDI. 


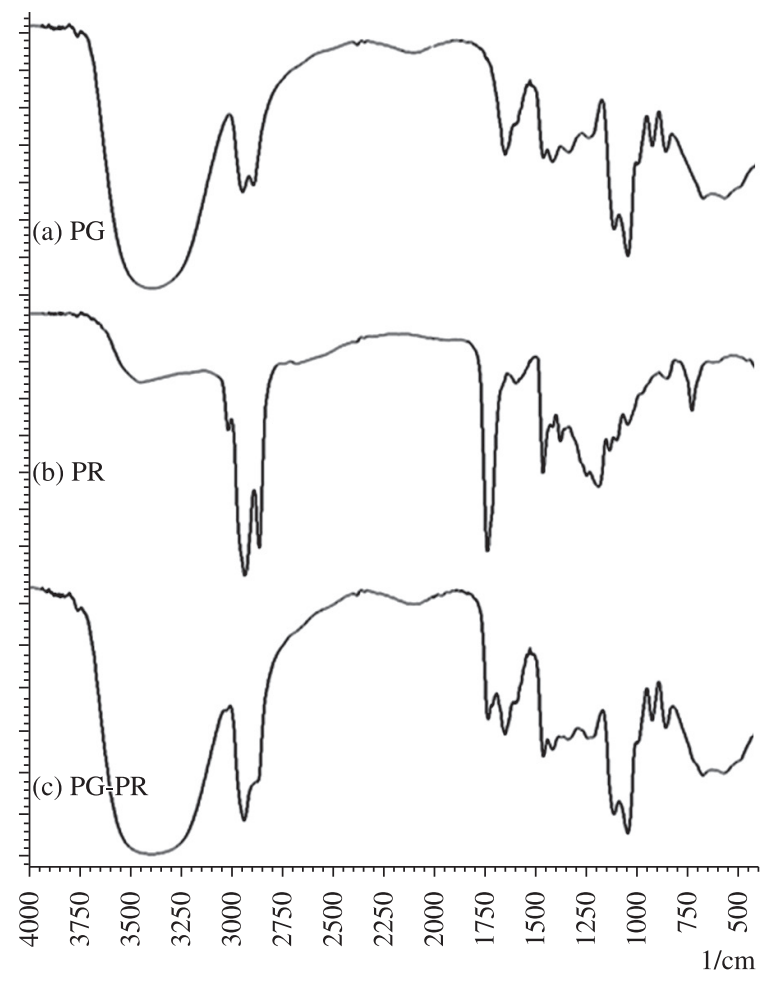

Figura 1. Espectros Infrarrojo de a) poliglicerol PG; b) Poliricinoleato PR; y c) Emulsificante poliglicerol-poliricinoleato PG-PR.

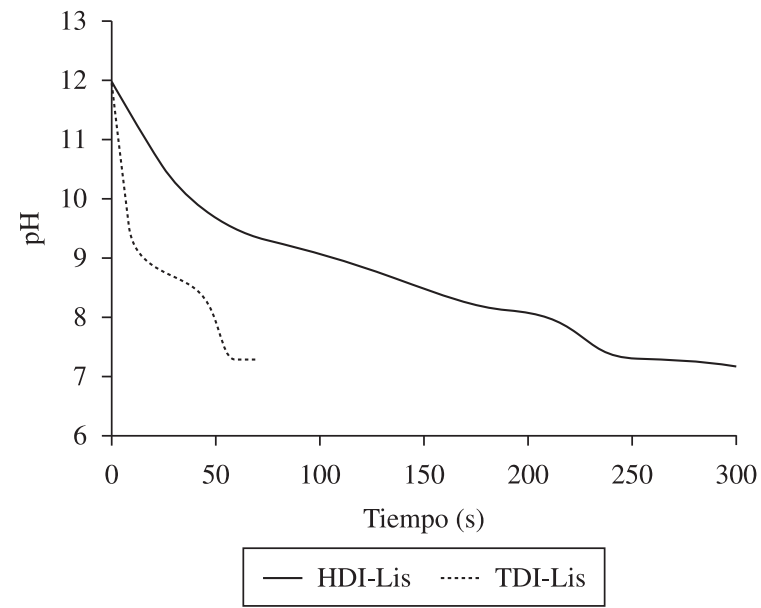

Figura 2. Seguimiento de la reacción en términos del valor de $\mathrm{pH}$. Condiciones de reacción: Índice $\mathrm{NCO}: \mathrm{OH}=1.25, \% \mathrm{PVA}=1 \%$, Span $80=0.1 \%$.

\section{Tipo de coloide protector}

Para analizar la influencia en el tamaño de partícula se evaluaron dos coloides protectores para la formación de la suspensión $\mathrm{O} / \mathrm{W}$, el alcohol polivinilico e hidroxietilcelulosa. Las condiciones de reacción fueron índice $\mathrm{NCO}: \mathrm{OH}=1.25$, Span $80=0.1 \%$, \%de coloide protector $=1 \%$. En el caso de utilizar hidroetilcelulosa no se formaron micropartículas, sino que se precipitó una película continua de poliurea, independiente del tipo de diisocianato, esto es debido a la hidrólisis que sufre la celulosa a $\mathrm{pH}$ tan altos y a la baja capacidad de absorción del mismo sobre las gotas de fase oleosa que permite coalescencia y aglomeración de las mismas, esto se muestra en la Figura 3 con una micrografía SEM de las partículas obtenidas.

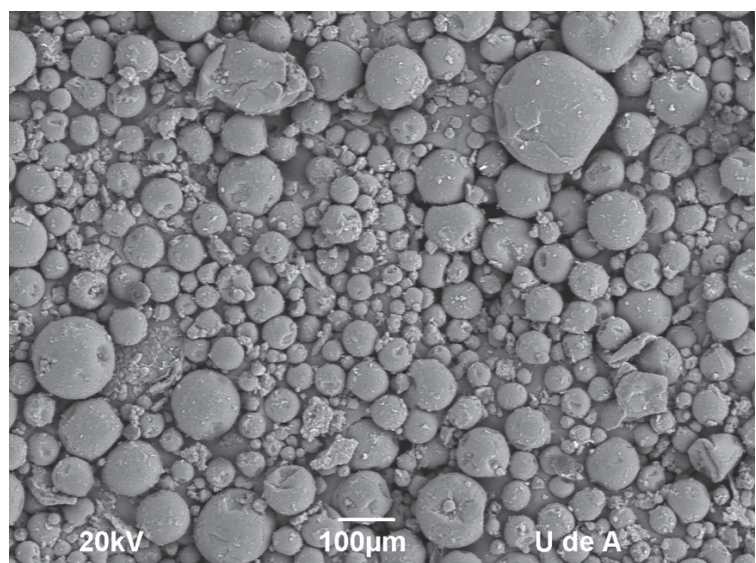

(a)

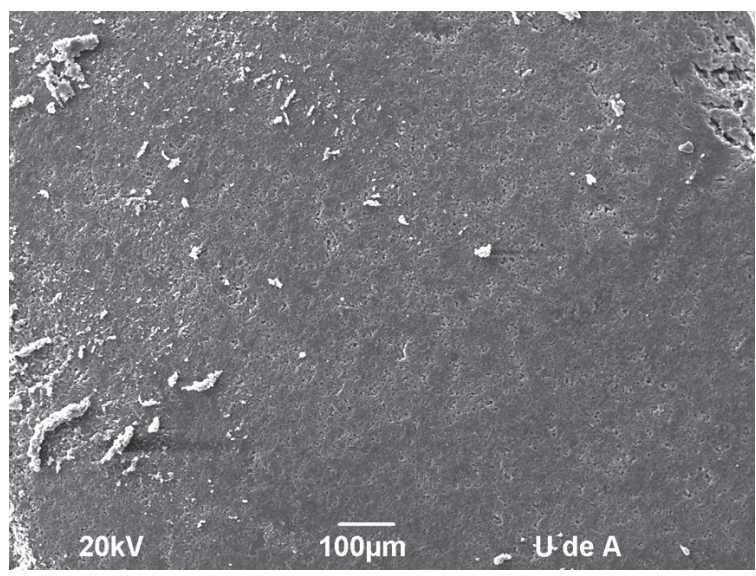

(b)

Figura 3. Micrografía SEM para el sistema HDI-Lis a) PVA y b) Hidroxietilcelulosa.

Tipo de emulsificante W/O

La Figura 4 muestra el espectro IR del perfume comercial donde se encuentran las señales correspondientes con grupos hidroxilo $\left(3388.9 \mathrm{~cm}^{-1}\right)$, carbonilo $\left(1733.21 \mathrm{~cm}^{-1}\right)$, estiramiento $-\mathrm{C}-\mathrm{O}-\mathrm{de}$ ácido, ester y éter (1238 a $\left.1046 \mathrm{~cm}^{-1}\right)$.

Para evaluar la necesidad de utilizar un emulsificante W/O, se determinó si hay reacción entre el diisocianato con el perfume para los dos sistemas HDI y TDI, a temperatura de $25{ }^{\circ} \mathrm{C}$, evaluando la desaparición del grupo NCO $\left(2270 \mathrm{~cm}^{-1}\right)$ por espectroscopia IR (Figuras 5 y 6) y se encontró que existen pequeñas diferencias entre los espectros realizados a 0 y 3 horas de reacción sin catalizador y existe formación de grupo uretano a $1728 \mathrm{~cm}^{-1}$, que se solapa con la señal del carbonilo del perfume, por lo cual es recomendable realizar la emulsificación W/O.

Se determinó el tamaño de partícula obtenido cuando se utilizan los dos emulsificantes SPAN80 Y PG-PRR, los resultados se muestran en la Figura 7.

Los mayores tamaños de partícula se obtienen paralas microcápsulas sin emulgente y con PG-PRR, debido a la mayor posibilidad de reacción del diisocianato con los hidroxilos libres del PG. La mezcla entre los dos emulgentes tendría un valor aproximado HLB de 2.65, que proporcionaría un valor medio de tamaño de partícula.

Las microcápsulas obtenidas con diisocianato aromático presentan menor tamaño de partícula, debido a su mayor reactividad.

Aunque la mezcla 0.5 SPAN 80:0.5 PG-PRR, no genera el menor tamaño de partícula, se selecciona para la optimización porque 


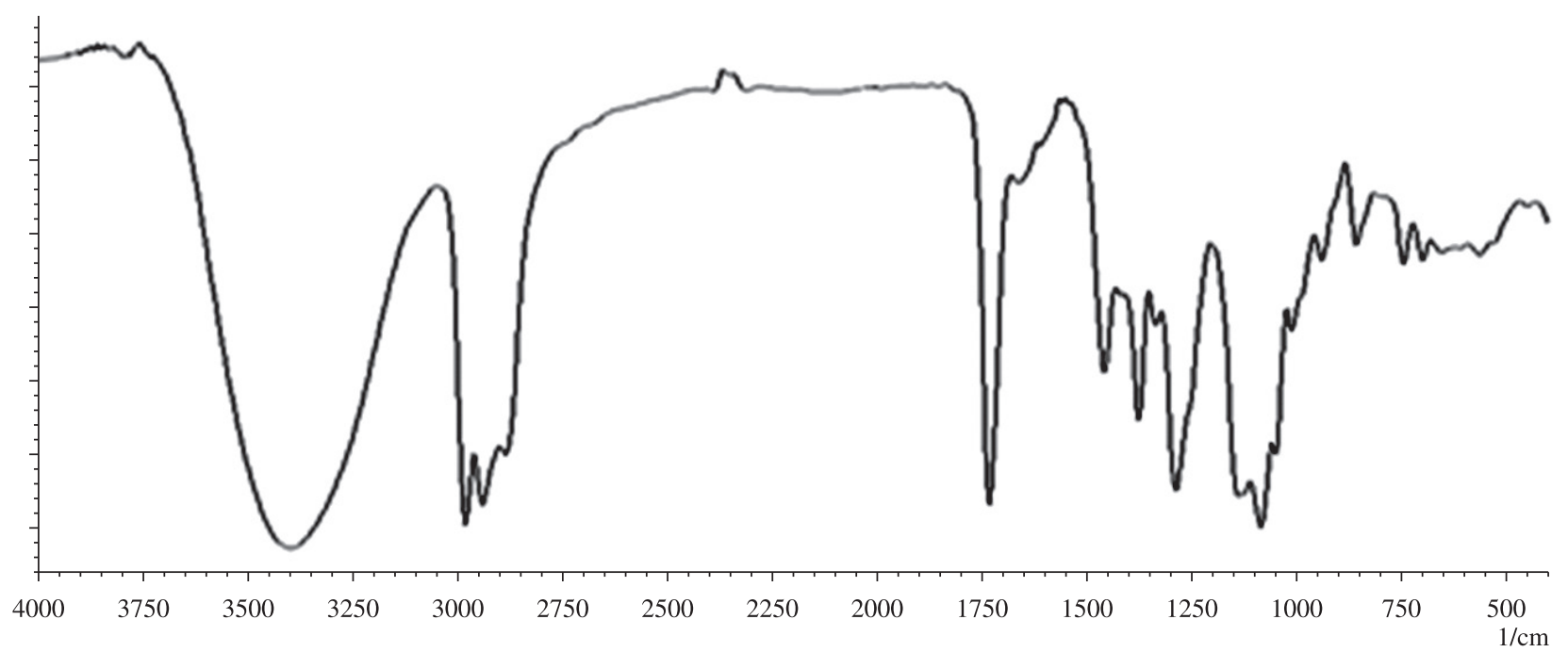

Figura 4. Espectro IR del perfume comercial.

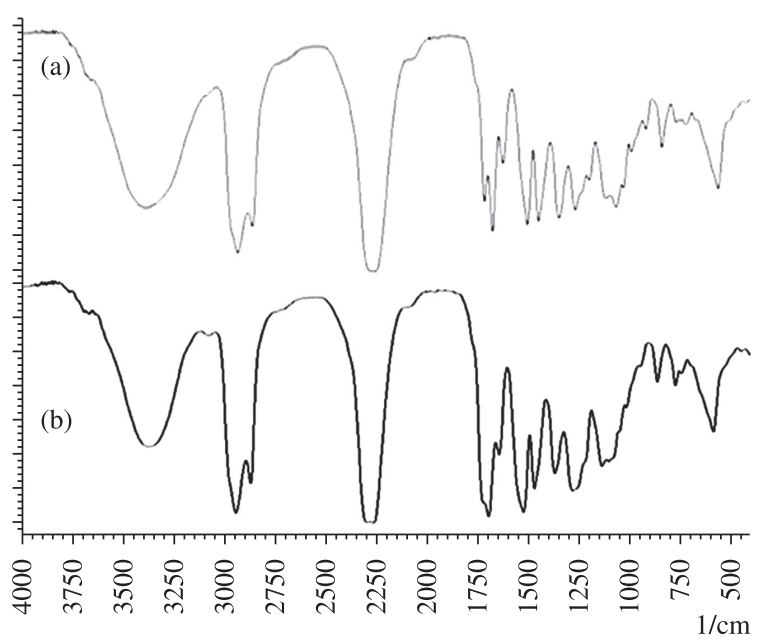

Figura 5. Espectro IR de a) Perfume-HDI tiempo: 0 horas y b) Perfume-HDI tiempo: 3 horas.

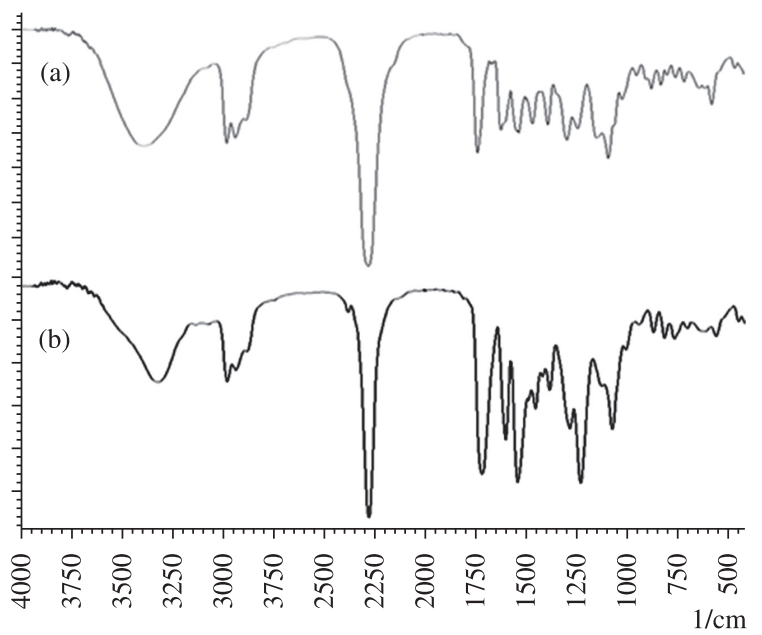

Figura 6. Espectro IR de a) Perfume-TDI tiempo: 0 horas y b) Perfume-TDI tiempo: 3 horas.

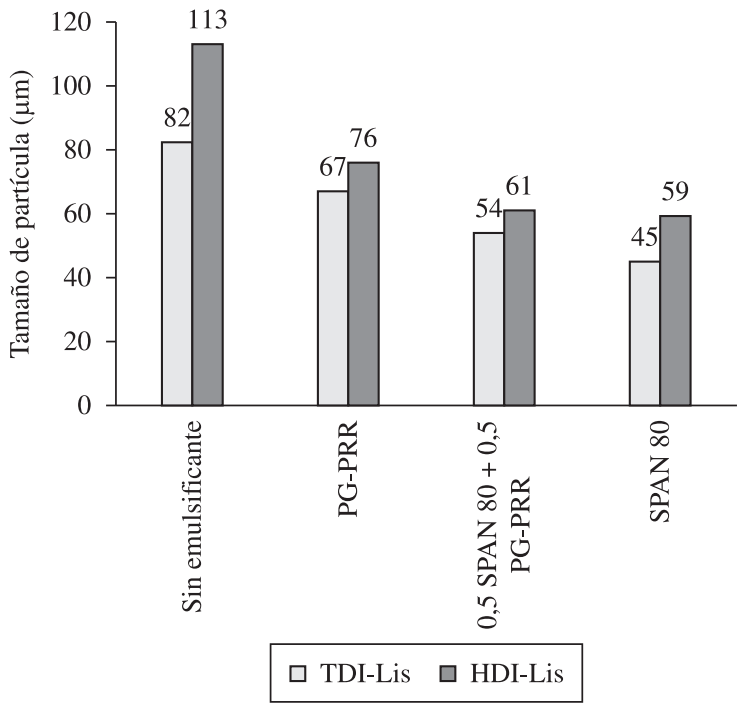

Figura 7. Tamaño de partícula $(\mu \mathrm{m})$ utilizando diferentes emulgentes $\mathrm{W} / \mathrm{O}$, las condiciones de reacción son: índice $\mathrm{NCO}: \mathrm{OH}=1.25, \% \mathrm{PVA}=1 \%$, cantidad de emulgente $=0.1 \%$.

disminuye costos del surfactante y aumenta la biodegradabilidad del mismo.

\section{Indice $\mathrm{NCO}: \mathrm{OH}$}

Se evalúa la influencia de la relación molar de grupos isocianato a grupo hidroxilo ó amina (NCO:OH), en tres niveles: 1, $1.25 \mathrm{y}$ 1.5. No hay diferencia significativa para los dos primeros niveles en el tamaño de partícula obtenido (Figura 8), para el índice de 1, las partículas son pegajosas y tienden a aglomerarse cuando se separan del medio acuoso, para evitar esto es requerido la adición de una cantidad mayor de diisocianato, porque puede reaccionar con los otros reactivos, incluso con el agua. Cuando el índice es de 1.5, el tamaño es mayor, debido a reacciones secundarias entre el diisocianato con los grupos amino que se forman en la capa exterior en la microcápsula, pero estas micropartículas presentan bajas propiedades mecánicas y se fracturan fácilmente por esfuerzos exteriores (Figura 9), esto es debido al aumento en los grupos urea que pueden segregarse y separarse de la red polimérica. 


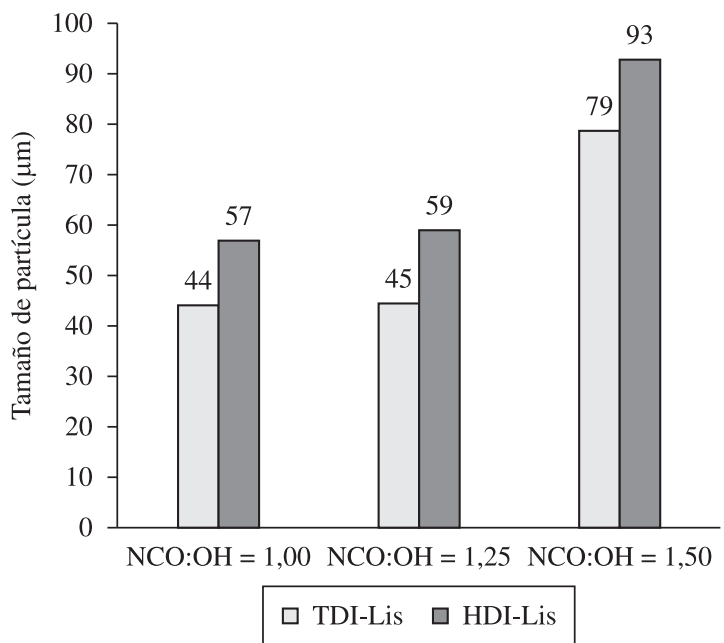

Figura 8. Tamaño de partícula $(\mu \mathrm{m})$ utilizando índice $\mathrm{NCO}: \mathrm{OH}$ de $1,1.25$ y 1.5 , las condiciones de reacción son: \%PVA $=1 \%$, SPAN $80=0.1 \%$.

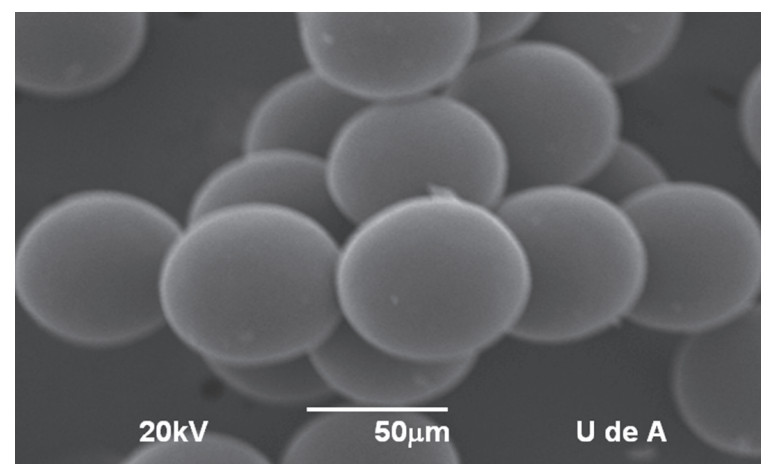

(a) Antes

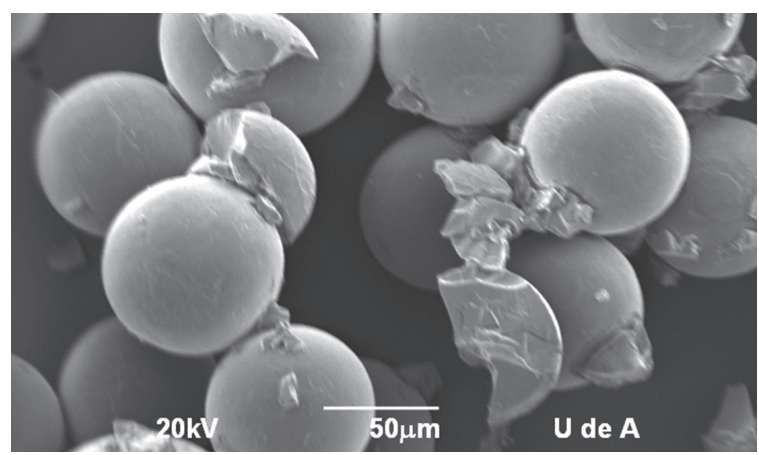

(b) Después

Figura 9. Micrografías de las microcápsulas HDI-Lis con índice de 1.5, antes y después de someter a agitación mecánica por 1 hora.
Síntesis y caracterización de las microcápsulas de poliurea

Después de encontrar la mejor relación entre las variables, se realiza la optimización del tamaño de partícula evaluando como factores la cantidad de agente emulsificante O/W (alcohol polivinilico) y de fase dispersa, basado en trabajos anteriores ${ }^{[18-19]}$

Los valores obtenidos para los experimentos y sus propiedades se describen en la Tabla 3.

Se utilizó el estadístico $t$ para cada una de las propiedades. Los $p$-valores mayores que 0.05 indican que no hay relaciones estadísticamente significativas entre las variables a un nivel de confianza del 95\%. Los valores obtenidos para los coeficientes con su respectivo p-valor se reportan en la Tabla 4.

Los términos que no son significativos de acuerdo a la prueba $\mathrm{t}$ (p-valor $>0.005$ ) se resaltan en la Tabla 4, la interacción de la relación en peso de las fases entre sí $\left(\mathrm{y}^{2}\right)$ y su interacción con él \% de alcohol polivinilico (xy) no son significativos. También se aprecia en el análisis de varianza que el modelo de regresión es significativo (p-valor $=0.000)$, y de acuerdo a los coeficientes de determinación $\mathrm{R}^{2}$ y $\mathrm{Raj}^{2}$ el modelo explica bien la variabilidad presente en los datos $\left(\mathrm{R}^{2} 95\right)$. También se aprecia el error estándar de estimación $(\mathrm{S})$ que dada la escala de medición de las variables de respuesta, tienen una magnitud relativamente pequeña.

La Figura 10 muestra las superficies de respuesta de los modelos obtenidos, donde puede verse que para minimizar el tamaño de partícula (z) para los dos sistemas estudiados se recomienda aumentar la cantidad de alcohol polivinílico (x) y disminuir la cantidad de fase dispersa (y), el nivel óptimo sería $\mathrm{x}=1, \mathrm{y}=-1$, correspondiente con \% PVA $=1.5, \mathrm{Wd} / \mathrm{Wc}=0.25$.

Tabla 3. Valores del tamaño de partícula de las microcápsulas.

\begin{tabular}{rrcc}
\hline $\mathbf{x}$ & $\mathbf{y}$ & $\begin{array}{c}\text { HDI-Lis } \\
(\boldsymbol{\mu m})\end{array}$ & TDI-Lis \\
\hline-1 & -1 & 40.73 & $\begin{array}{c}\text { Tamoñ de partícula } \\
(\boldsymbol{\mu m})\end{array}$ \\
1 & -1 & 12.67 & 36.57 \\
0 & 1 & 55.74 & 10.58 \\
1 & 0 & 20.91 & 51.63 \\
0 & -1 & 35.84 & 15.71 \\
1 & 1 & 37.93 & 33.45 \\
0 & 0 & 45.81 & 25.37 \\
-1 & 1 & 62.63 & 43.67 \\
-1 & 0 & 56.45 & 54.81 \\
0 & 0 & 51.34 & 45.63 \\
0 & 0 & 50.75 & 45.34 \\
0 & 0 & 49.89 & 39.47 \\
0 & 0 & 45.45 & 42.35 \\
0 & 0 & 53.39 & 38.39 \\
\hline
\end{tabular}

Tabla 4. Coeficientes obtenidos para las superficies de respuesta.

\begin{tabular}{|c|c|c|c|c|c|c|c|c|}
\hline & $\begin{array}{c}\text { Constante } \\
\text { p-valor }\end{array}$ & $\begin{array}{c}\mathbf{x} \\
\text { p-valor }\end{array}$ & $\begin{array}{c}\mathbf{y} \\
\text { p-valor }\end{array}$ & $\begin{array}{c}\mathbf{x}^{2} \\
\text { p-valor }\end{array}$ & $\begin{array}{c}\mathbf{y}^{2} \\
\text { p-valor }\end{array}$ & $\begin{array}{c}\text { xy } \\
\text { p-valor }\end{array}$ & $\begin{array}{c}\mathbf{R}^{2} \\
\text { Raj }^{2} \\
\text { S }\end{array}$ & $\begin{array}{c}\text { Análisis varianza } \\
\text { p-valor }\end{array}$ \\
\hline \multirow[t]{3}{*}{ HDI-Lis } & 49.0315 & -14.7167 & 11.1767 & -9.1309 & -2.0209 & 0.8400 & 96.35 & 0.000 \\
\hline & 0.000 & 0.000 & 0.000 & 0.002 & 0.343 & 0.632 & 94.06 & \\
\hline & & & & & & & 3.3755 & \\
\hline \multirow{2}{*}{ TDI-Lis } & 0.000 & 0.000 & 0.000 & 0.001 & 0.958 & 0.650 & 92.22 & \\
\hline & & & & & & & 3.6549 & \\
\hline
\end{tabular}




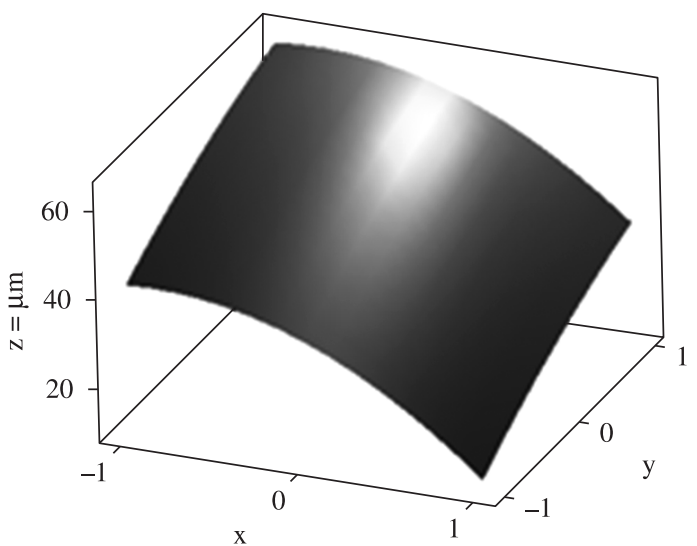

(a) Superficie HDI-Lis

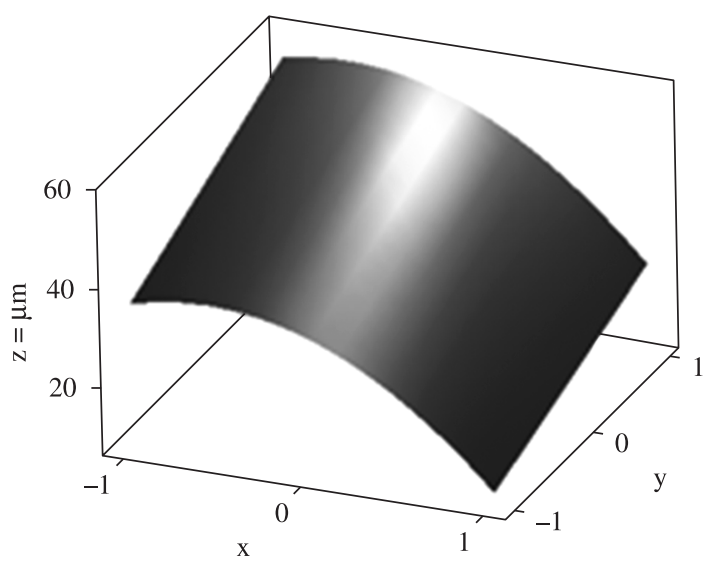

(c) Superficie TDI-Lis

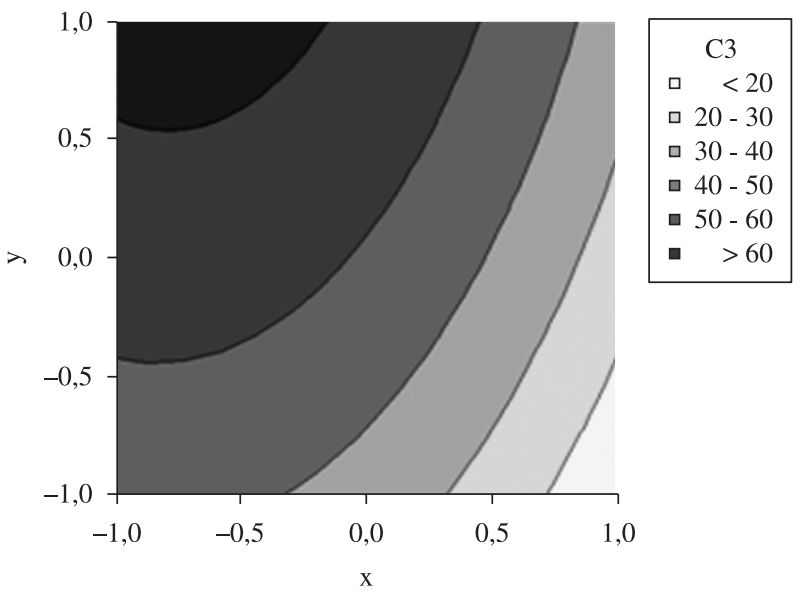

(b) Contorno HDI-Lis
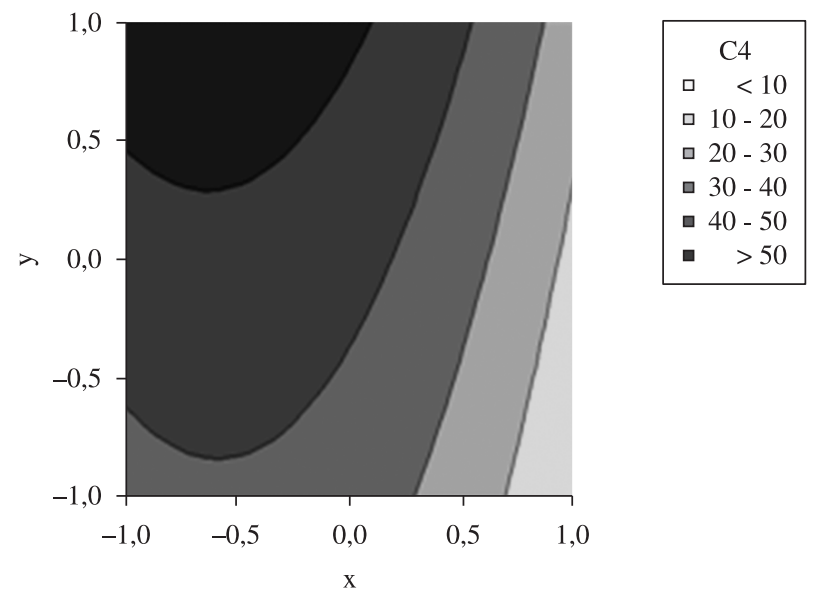

(d) Contorno TDI-Lis

Figura 10. Superficies de respuesta y gráficas de contorno para: a) y c) Superficie de respuesta para los sistemas HDI-Lis y TDI-Lis, respetivamente; b) y d) Gráfico de contorno para los sistemas HDI-Lis y TDI-Lis, respetivamente. Donde z: variable de respuesta tamaño de partícula ( $\mu$ m), x: variable independiente codificada $\%$ alcohol polivinilico, y: variable codificada correspondiente a la relación en peso de la fase dispersa a la fase continua.

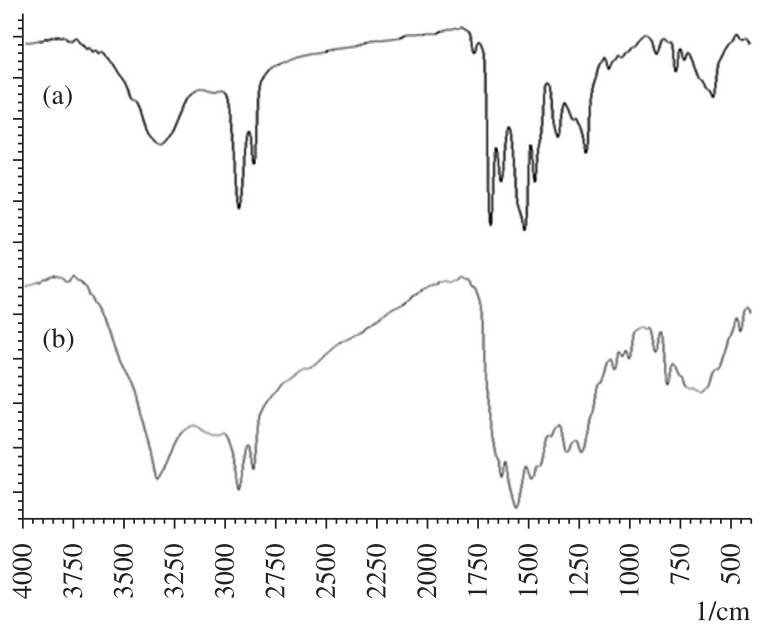

Figura 11. Espectro IR de las microcápsulas vacias de poliurea a) HDI-Lis y b) TDI-Lis.

Con base en los resultados obtenidos se prepararon microcápsulas y se caracterizaron con las siguientes condiciones: $\mathrm{NCO}: \mathrm{OH}=1.25$, 0.5 SPAN 80:0.5 PG-PRR $=0.1 \% ; \% \mathrm{PVA}=1.5 ; \mathrm{Wd} / \mathrm{Wc}=0.25$.
La Figura 11 muestra el espectro IR para la poliurea obtenida de HDI-Lis y TDI-Lis, sin perfume, donde las principales señales son: la banda de absorción cerca de $3320 \mathrm{~cm}^{-1}$ es asignada a $\mathrm{NH}$, y la banda cerca a $1640 \mathrm{~cm}^{-1}$ corresponde con el carbonilo de urea. No se observa la banda correspondiente al grupo isocianato en 2200-2280 $\mathrm{cm}^{-1}$. Para el espectro IR de HDI-Lis, aparece un pequeño pico en $1763 \mathrm{~cm}^{-1}$, correspondiente con grupos uretano, formados por la reacción de algunos grupos isocianato con hidroxilos, debido a la baja reactividad del diisocianato alifático.

La superficie de las microcápsulas obtenidas con TDI presentan mayor rugosidad y sus paredes parecen ser más gruesas, esto es debido a la mayor reactividad de este diisocianato, como se muestra en las fotografías SEM de la Figura 12.

La poliurea es un termoplástico con una alta temperatura de fusión alrededor de $285^{\circ} \mathrm{C}$, el análisis de calorimetría diferencial de barrido a menor temperatura para el perfume y las microcápsulas obtenidas, se muestra en la Figura 13 y permite evidenciar un pico entre 87 y $130{ }^{\circ} \mathrm{C}$, debido a la ebullición endotérmica del perfume comercial.

El análisis de DSC permite cuantificar la cantidad de perfume microencapsulado, mediante la relación del calor de evaporación del perfume puro y el calor obtenido después de microencapsular. Los valores se reportan en la Tabla 5. 


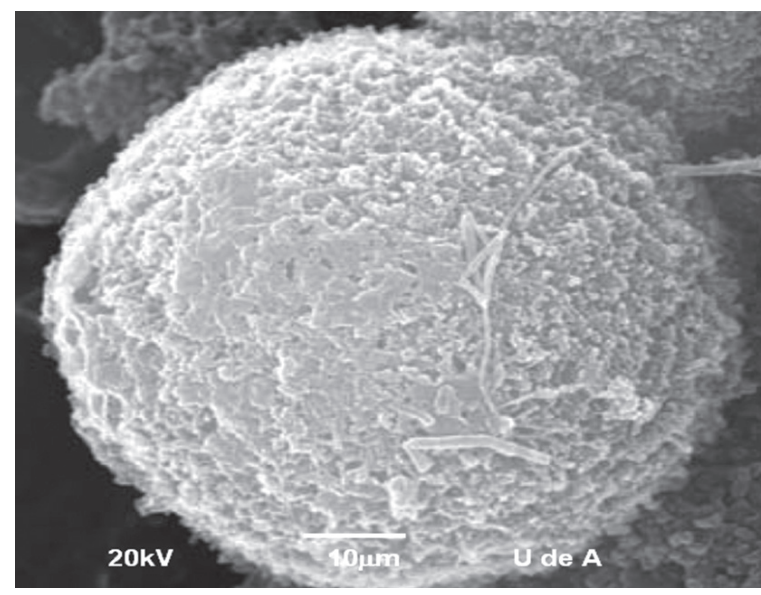

(a) TDI-Lis

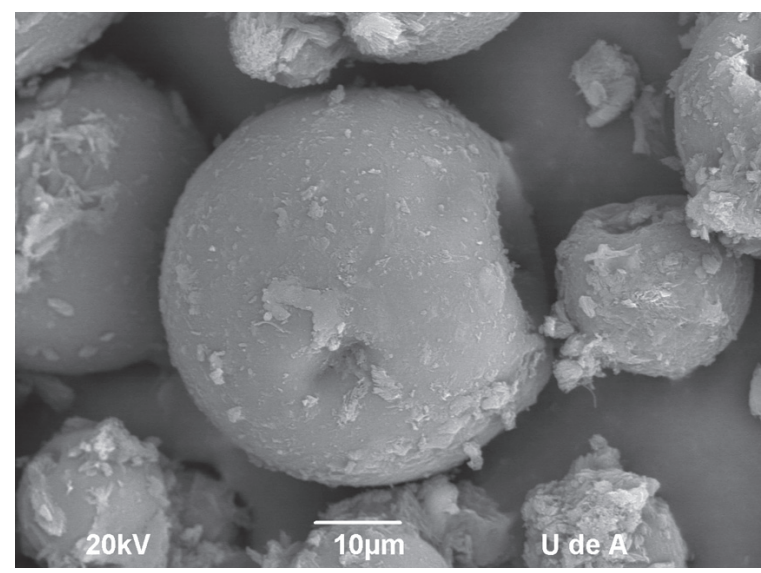

(b) HDI-Lis

Figura 12. Micrografías de las microcápsulas a) TDI-Lis y b) HDI-Lis.

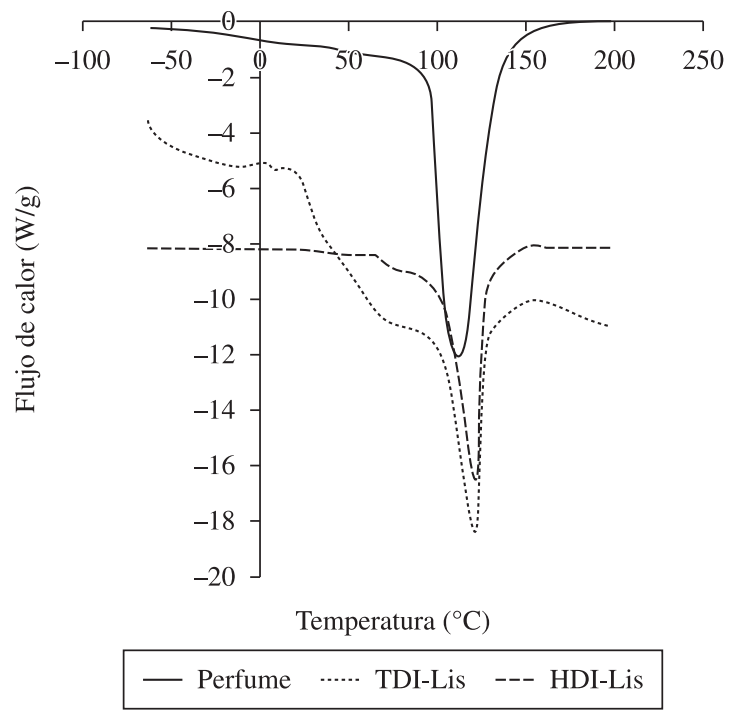

Figura 13. Análisis de calorimetría diferencial de barrido (DSC) para el perfume, microcápsulas de HDI-Lis y TDI-Lis.

De acuerdo con la formulación el sistema TDI-Lis tiene $44 \%$ perfume y el HDI-Lis tiene $45 \%$ de perfume, de acuerdo con los resultados obtenidos mediante DSC, podemos ver que la pérdida final de perfume es de 13 y $16 \%$ respectivamente, debido a volatilización del mismo.
Tabla 5. Calor de evaporación obtenido por DSC del perfume y micropartículas de HDI-Lis y TDI-Lis.

\begin{tabular}{lccc}
\hline Muestra & $\begin{array}{c}\text { Calor de evaporación } \\
\left(\text { J.g }^{-1}\right)\end{array}$ & \%Perfume & \% Poliurea \\
\hline Perfume & 175.3 & 100.0 & 0.0 \\
TDI-Lis & 669.8 & 38.2 & 61.8 \\
HDI-Lis & 662.5 & 37.8 & 62.2 \\
\hline
\end{tabular}

\section{Conclusiones}

La microencapsulación de perfume comercial hidrofílico se realiza mediante la formación de una coraza de poliurea, obtenida por la reacción de diisocianato aromático y alifático, con Lisina, utilizando una doble emulsificación y polimerización interfacial. Se requiere $\mathrm{pH}$ por encima de 12 para favorecer la amina libre, se recomienda el uso de alcohol polivinilico como coloide protector y como emulsificante W/O puede emplearse una mezcla 50:50 de Span 80 y PG-PR, para obtener tamaños medios de partícula, el índice $\mathrm{NCO}: \mathrm{OH}$ recomendado es de 1.25, para evitar coalescencia de las partículas y bajas propiedades mecánicas.

La optimización mediante superficie de respuesta permite obtener unos niveles adecuados para la síntesis, \%PVA: 1.5 y $\mathrm{Wd} / \mathrm{Wc}=0.25$.

Se presentan pérdidas de perfume entre un 13 y $16 \%$ empleando doble emulsificación, las microcápsulas obtenidas con TDI tienen menor tamaño de partícula, más rugosidad y mejor resistencia térmica que las de HDI.

\section{Referencias}

1. Fragiadakis, D.; Gamache, R.; Bogoslovov, R. B. \& Roland, C. M. Polymer., 51, p.178 (2010).

2. Sopeña, F.; Maqueda, C. \& Morillo, E. - Cienc. Investig. Agr., 35, p.27 (2009).

3. Kleban, M.; Klug, G. \& Weisser, J. - "Oficina española de patentes y marcas", ES 2267645 T3 (2003).

4. Li, G.; Feng, Y.; Gao, P \& Li, X. - Polym. Bull., 60, p,725 (2008).

5. Gao, G.; Qian, C. \& Gao, M. - Chin. Chem. Lett., 21, p.533-537 (2010).

6. Ji, H.; Kuang, J. \& Qian, Y. - Catal. Today, 105, p.605 (2005).

7. Ley, S.; Mitchell, C.; Pears, D.; Ramarao, C.; Yu, J. \& Zhou, W. - Org. Lett., 5, p.4665 (2003).

8. Girauda, S.; Bourbigota, S.; Rocherya, M.; Vromana, I.; Tighzertb, L.; Delobelc, R. \& Poutchd, F. - Polym. Degrad. Stab., 88, p.106 (2005).

9. Cho, J.; Kwon, A. \& Cho, C. - Colloid Polym. Sci., 280, p.260 (2002).

10. Liang, C.; Lingling, W.; Hongbo, S. \& Zhibin, Z. - Energy Convers. Manag., 50, p.723 (2009).

11. Zhang, H. \& Wang, X. - Sol. Energy Mater. Sol. Cells, 93, p.1366 (2009).

12. McIlroy, D.; Blaiszik, B.; Caruso, M.; White, S.; Moore, J. \& Sottos, N. - Macromolecules, 43, p.1855 (2010).

13. Villamizar, L. \& Martínez, F. - Rev. Fac. Quím. Farm., 15, p.123 (2008).

14. Martín, M. J.; Morales, M. E.; Gallardo, V. \& Ruiz, M. A. - Ars Pharm., 50, p.43 (2009).

15. Dhumal, S. S. \& Wagh, S. J. - J. Membr. Sci., 325, p.758 (2008). 16. Ni, P.; Zhang, M. \& Yan, N. - J. Membr. Sci., 103, p.51 (1995). 17. Wilson, R.; Schie, V. \& Howes, D. - Food Chem. Toxicol., 36, p.711 (1998). 18. Kobaslija, M. \& McQuade, T. - Macromolecules, 39, p.6371 (2006).

19. Rodrigues, S.; Fernandes, I.; Martins, I.; Mata, V.; Barreiro, F. \& Rodrigues, A. - Ind. Eng. Chem. Res., 47, p.4142 (2008).

Enviado: $20 / 04 / 10$

Reenviado: $26 / 08 / 10$ Aceito: $22 / 09 / 10$

DOI: $10.1590 / \mathrm{S} 0104-14282011005000030$ 